\title{
Association Between Neighborhood's Visual Quality and Resident's Physical Activity: A Case Study
}

Shahrzad Parto

* PhD Researcher in Urban Design, Faculty of Art and Architecture, Bu-Ali Sina University, Hamadan, Iran.(Corresponding author)

parto.shahrzad@gmail.com

Mehrdad Karimimoshaver

Assistant Professor, Dept. of Urban Design, Faculty of Arts and Architecture, Bu-Ali Sina University, Hamadan, Iran

Mohammad-Saeed Izadi

Associate Professor, Dept. of Architecture, Faculty of Arts and Architecture, Bu-Ali Sina University, Hamadan, Iran

Rouhollah Zaboli

Associate Professor, Dept. of Health Services Administration, Faculty of Health, Baqiyatallah University of Medical Sciences, Tehran, Iran

$\underline{x}$

Received: 2021/04/05

Accepted: 2021/06/28

Doi:10.52547/ijhehp.9.3.269

\section{ABSTRACT}

Background and Objective: The visual quality of the environment affects the level of physical activity of citizens, so it is necessary to take basic steps to reduce the gap between urban planning and public health through interdisciplinary studies. This study aimed to identify the relationship between visual quality and amount of physical activity in the neighborhood Mehrshahr of Karaj.

Materials and Methods: This study was a quantitative research that was done with a confirmatory factor analysis approach. The statistical population was 100 residents of Mehrshahr neighborhood of Karaj who were randomly selected. Data were collected using two questionnaires. Data analysis and hypothesis testing by confirmatory factor analysis (CFA) and partial least squares (PLS) tests, Spearman and analysis of variance were one-way.

Results: The results showed that the quality of neighborhood $s$ visual quality with mean and standard deviation was $3 / 62 \pm 0 / 57$ and there was a statistically significant difference with the average population $(p=0 / 001)$. The mean and standard deviation of residents' physical activity was $3.24 \pm 0.51$ which was statistically significant $(P=0.001)$. The results of path analysis showed that the quality of visual perception of residents of the environment has a positive and significant effect on the level of physical activity of residents in the environment. Improving visual diversity, becoming more regular, opening and expanding the environment, being more natural, better preservation, the presence of historical elements in the environment and its freshness and vibrancy, in the eyes of residents leading to physical activity and walking, cycling, Intense and moderate physical activity will be in the environment.

Conclusion: Improving the visual quality of the environment can facilitate and encourage participation in physical activities, and to improve the physical activity of citizens and improve public health, changes and improvements in some visual qualities will have a greater impact on the health of citizens. Therefore, in order to increase the level of physical activity of citizens, it is possible to intervene purposefully and accurately in the body of the environment.

Keywords: Aesthetic Quality, Physical Activity, Public Health, Residential Neighborhood. Paper Type: Research Article.

Citation (Vancouver): Parto Sh, Karimimoshaver M, Izadi M, Zaboli R. Association Between Neighborhood's Visual Quality and Resident's Physical Activity: A Case Study. Iran J Health Educ Health Promot. Autumn 2021;9(3): 269-279.

- Citation (APA): Parto Sh., Karimimoshaver M., Izadi M., Zaboli R. (Autumn 2021). Association Between Neighborhood's Visual Quality and Resident's Physical Activity: A Case Study. Iranian Journal of Health Education \& Health Promotion., 9(3), 269-279. 


\section{ارتباط ميان كيفيت بصرى محيط شهرى و ميزان فعاليت بدنى شهروندان: \\ يك مطالعه موردى}

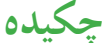

زمينه و هدف : با توجه به تأثير جشمخير كيفيت بصرى محيط بر ميزان فعاليت بدنى شهروندان ضرورى دان

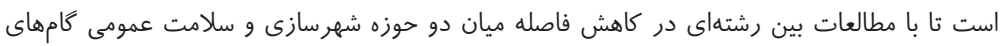

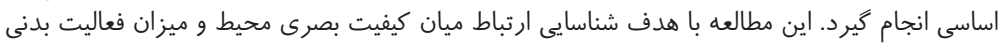

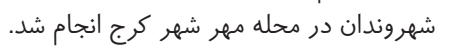

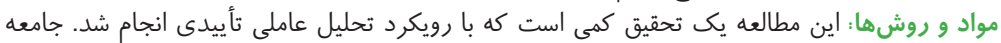

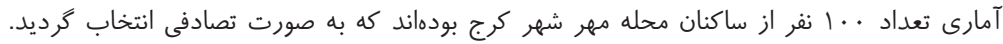

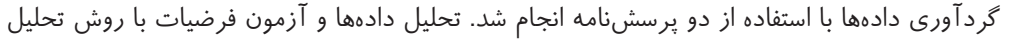

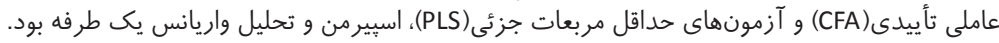

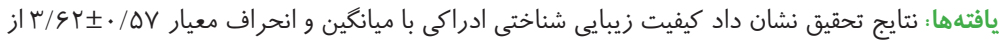

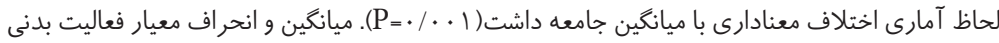

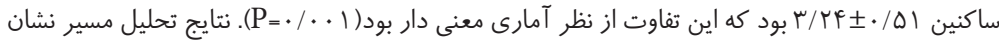

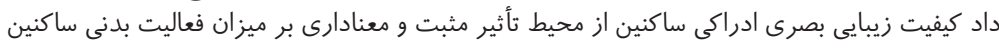

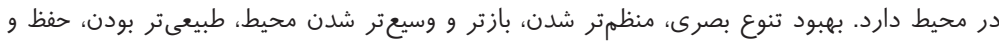

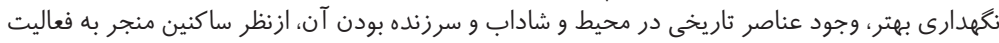

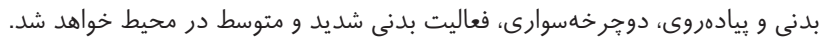

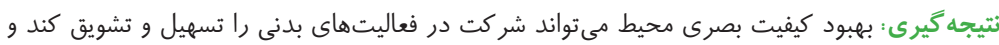

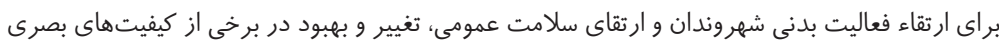

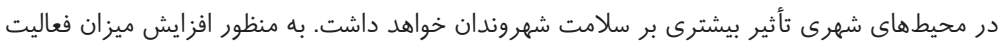

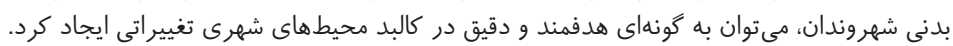

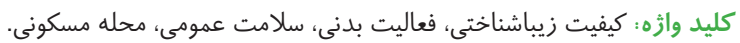
نوع مقاله : مطالعه يزوهشى.

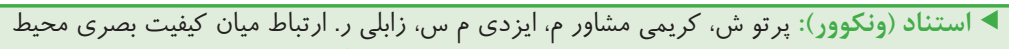

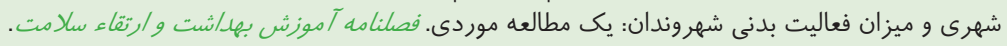

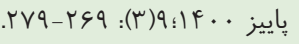

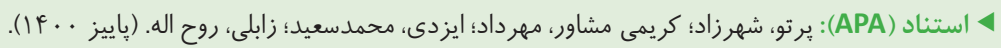

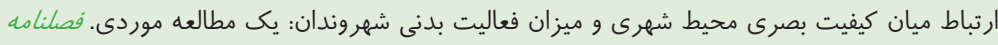

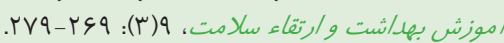

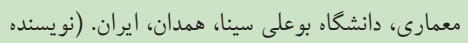
parto.shahrzad@gmail.com دانشيار، كروه طر احى شهرى، دانشكده هنر و معمارى، دانشكاه بوعلى سينا، همدان، ايران

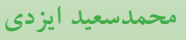

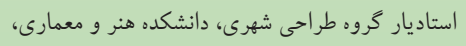
دانشاء بوعلى سينا، همدان، ايران روح اله زابلى داني دانشيار، كروه مديريت خداري خدات بهداشتى، دانشكده بهداشت، دانشكاء علوم يز شكى بقيه اله، تهران، ايران 
متمر كز بودهاند. اين رويكرد امروزه مورد انتقاد قرار كرفته جرا كه تأكيد بيش از حد بر ويث كى هاى فردى دارد و بستر شكل گيرى رفتار

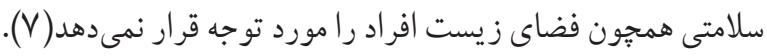

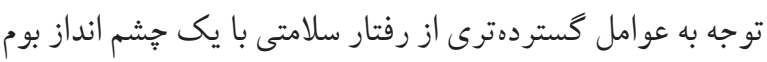

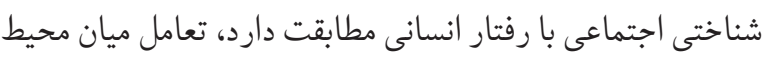
كالبدى، اجتماعى و فردى و نياز به حداكثر ساختن ساز كارى انسان

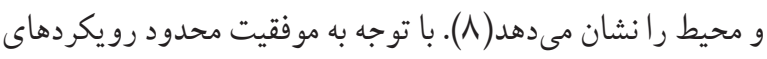

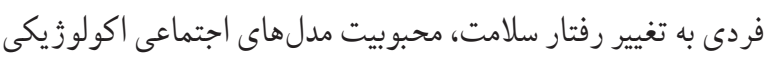

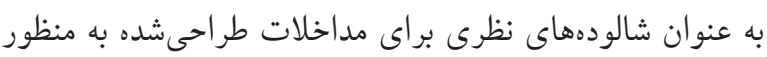

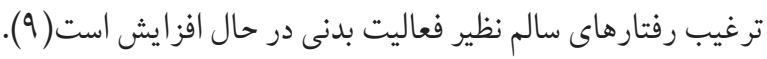
مطالعات متعددى به تعيين روابط كليدى بين بافت شهرى و ورئر

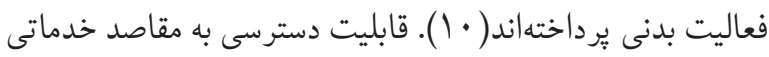

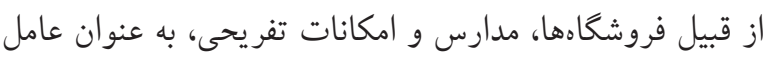

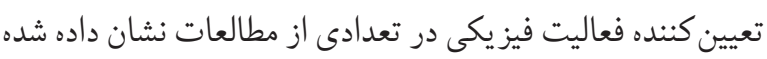
است(1) (1). برخى شواهد وجود دارد كه سطوح فعاليت فيزيكى انفرادى، با تعداد امكانات ورزشى در دسترس افزايش مى يابد و استفاده از

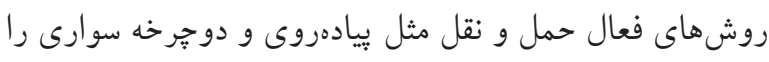

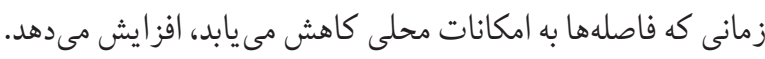

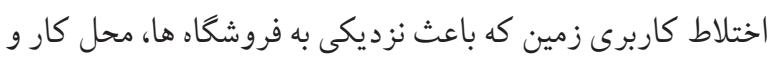

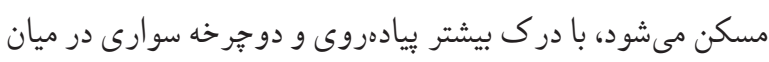

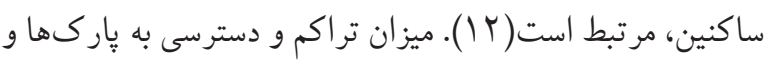

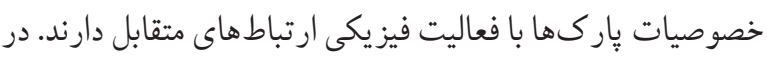

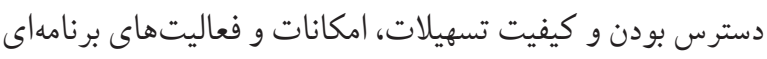

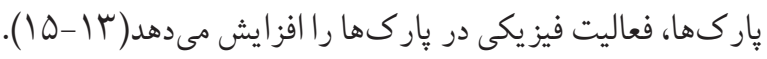

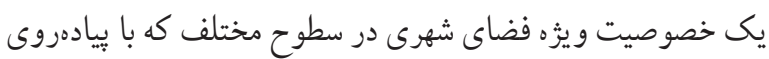

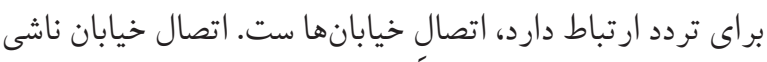
از مستقيم بودن و در دسترس بودن راههاى گزينهاى بين خانه و

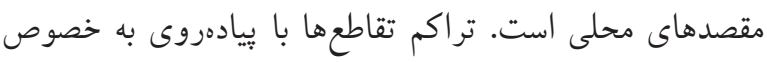

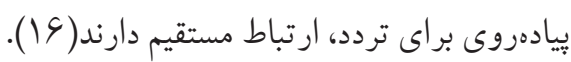

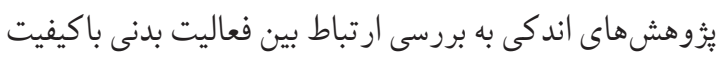

بيمارىهاى غير واگير هر ساله در جهان منجر به مرگ حدود وس

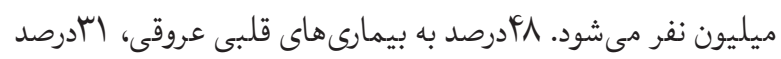

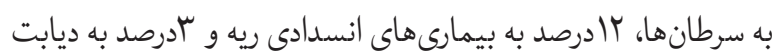

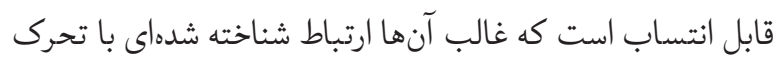

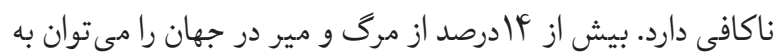
فعاليت بدنى ناكافى نسبت داد، ضمن آن كه اين رفتار در ايجاد

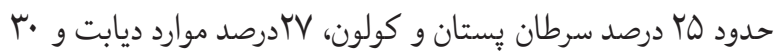
درصد از موارد بيمارىهاى قلبى عروقى نقش دارد( (1). در ايران نيز فعاليت بدنى ناكافى از عوامل خطر جدى بيمارىهاى غير واكير عرودي

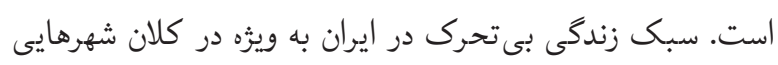

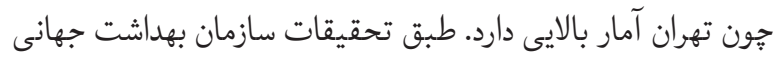

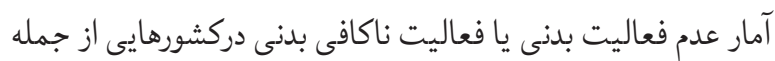
آمريكاو منطقه مديترانه شرقى بالاتر از مناطق ديخر بوده است(ب (Y).

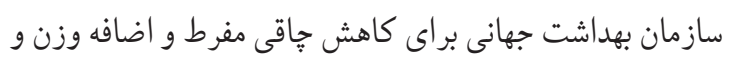

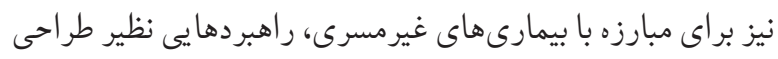

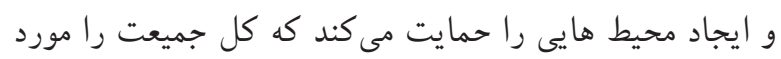

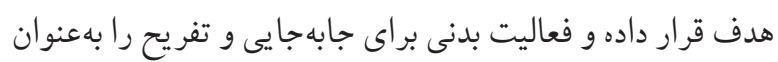

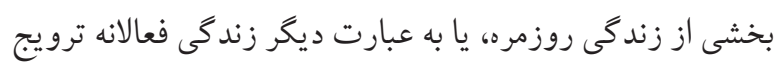

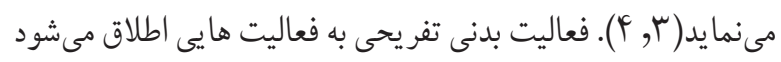

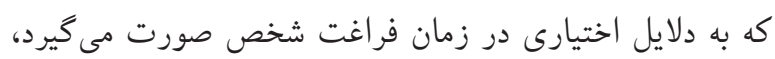

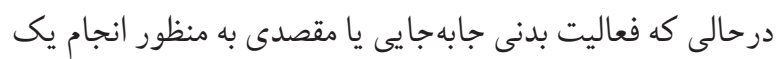

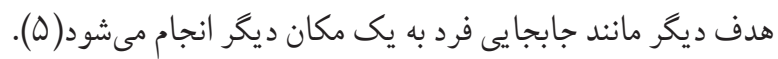

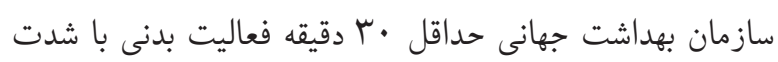

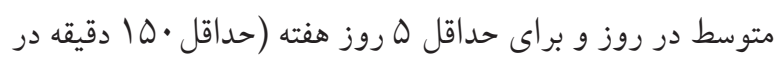

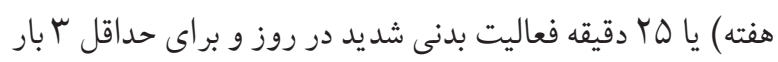

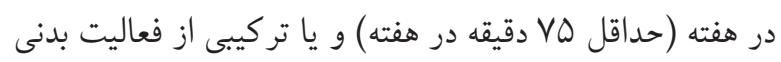
متوسط و شديد با نسبت هاى متفاوت را به عنوان حداقل فعاليت

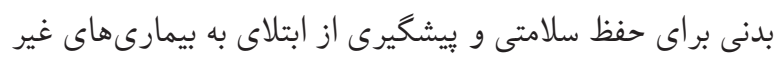

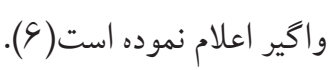

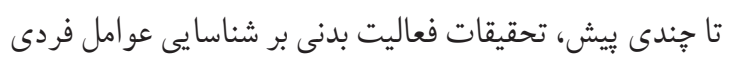


بصرى و ويزگ هاى زيبايى شناختى محيط بر رفتار فعاليت بدنى ساكنان در محله مهرشهر كرج بوده است.

مو ادو ورش

اين مطالعه يك تحقيق كمى است كه در سال 99 با رويكرد تحليل

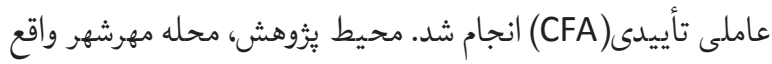

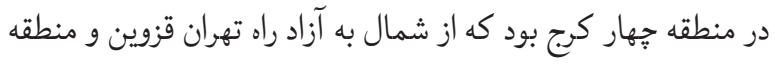

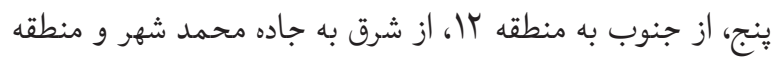

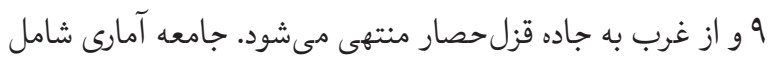
ساكنين در اين منطقه بود. با توجه به براكندگى و تعداد زياد ساكنين

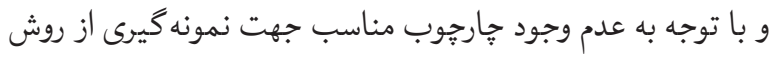
نمونه گيرى در دسترس استفاده شد. معيار اصلى ورود به مطالعه

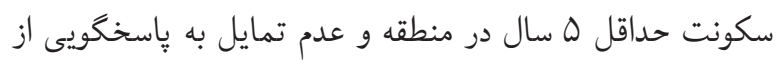
معيارهاى خروج به مطالعه بود. در تحليل عاملى تائيدى حداقل حجم نمونه بر اساس عاملها تعيين مى شود. در مدل يابى معادلات

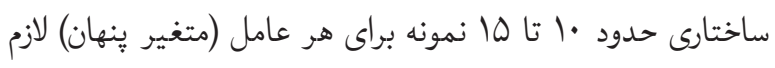

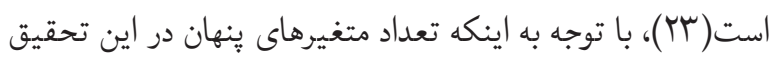

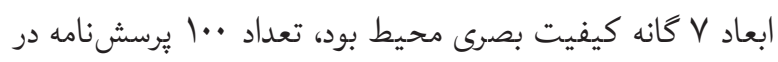

$$
\text { اختيار ساكنين قرار كرفت. }
$$

بهمنظور جمع آورى دادههاى تحقيق نيز از دو يرسشنامه استفاده شد. يرسشنامه اول محقق ساخته بود كه بر اساس مطالعه مرور سيستماتيك كه توسط نو يسند كان انجام شد طراحى كرديد (19 19). اين يرسش داراى دو بخش بود. بخش اول ويز كىهاى فردى و

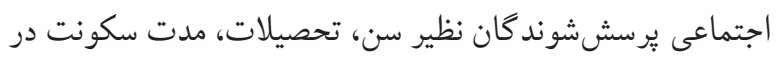

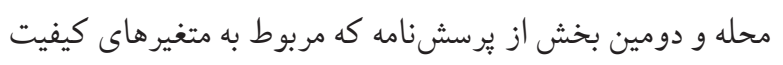

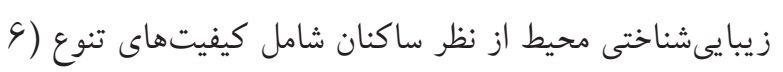

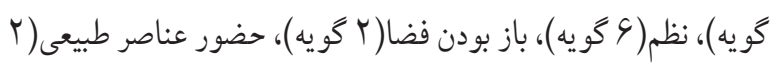

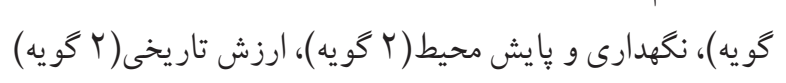

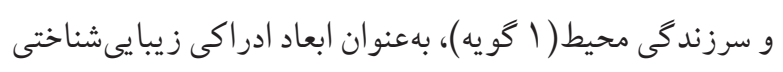

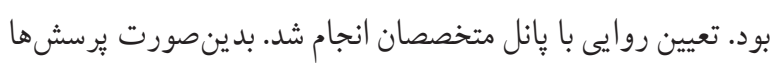

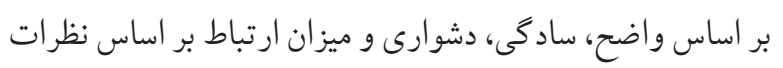

بصرى محيط يُرداختهاند. اين در حالى است كه محيط كالبدى و كيفيت زيبايى شناسى آن تأثيرات مهمى بر تجربه فردى در آن دآن دارد.

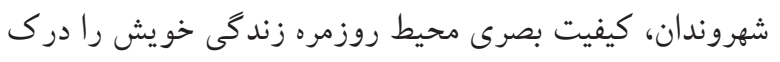

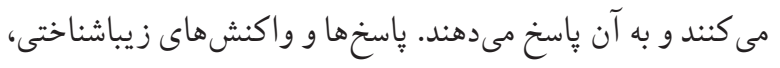
از واكنشهاى ضرورى انسانى است كه توسط محيط بر انخيخته مى گردد) (IV). ارزيابى كيفيت بصرى محيط از ديرباز مورد توجه معماران و ودان طر احان شهرى بوده است. تحقيق در مورد كيفيت زيبايى شناختى و

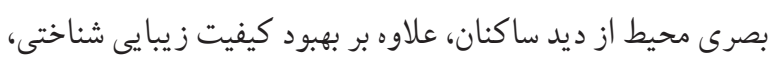

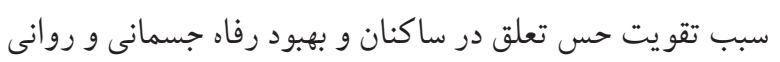

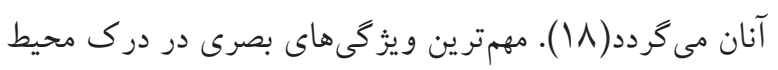

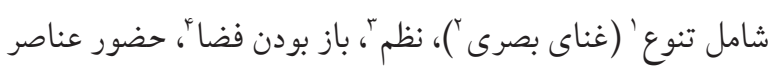

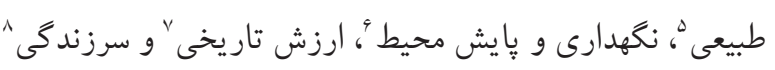

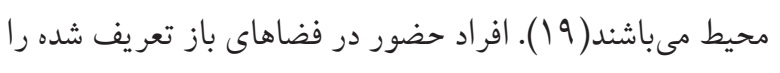

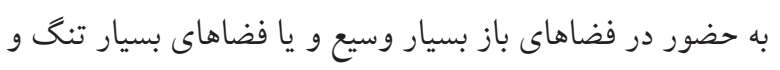

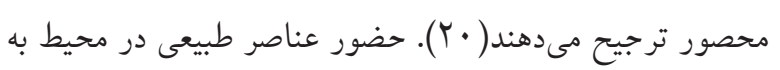

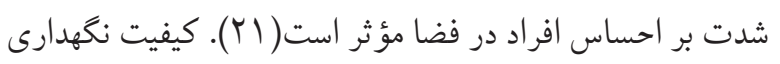

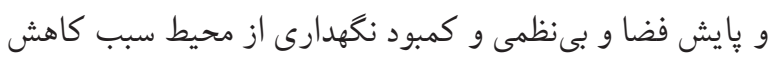

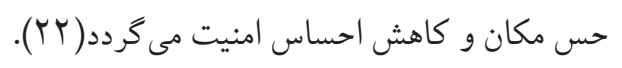
با توجه به تأثير عناصر بصرى و كيفيت و زيبايى احناسى محيط

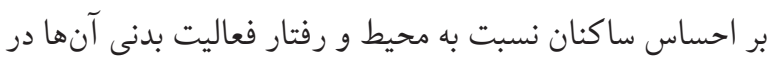

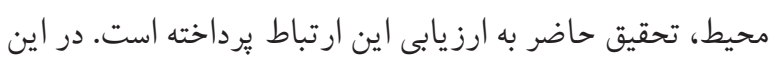
تحقيق و يز گىهاى بصرى و كيفيت زيبايى شناختى محيط به عنوان

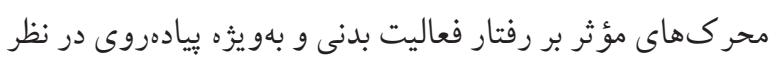

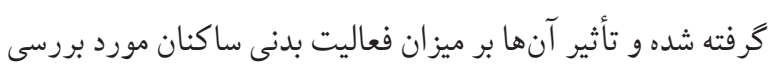
قرار كرفت. هدف اصلى اين تحقيق بررسى ارتباط و تأثير كيفيت

\footnotetext{
1. Diversity

2. Visual richness

3. Order

4. Openness

5. Naturalness

6. Upkeep

7. Historical significance

8. Livability
} 
جزئى ‘ استفاده شد. جهت تحليل ساختار درونى برسشنامه و تعيين

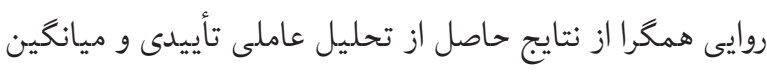

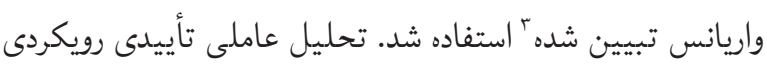
مدل يابى براى مطالعه سازه هاى فرضى است. زمانى كه ساختار

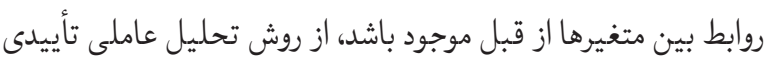

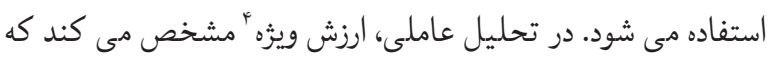

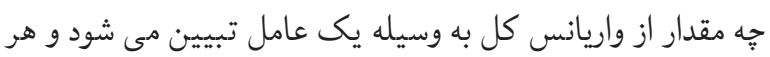

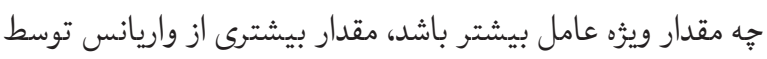

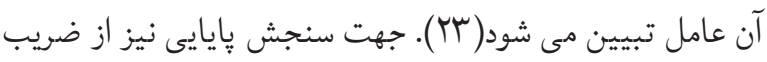

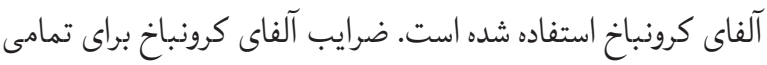

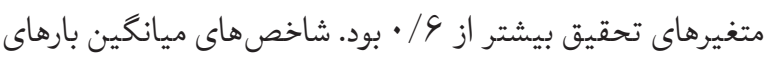

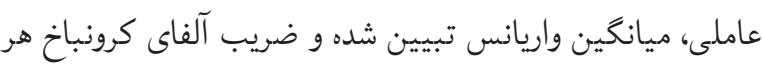
يك از متغيرهاى تحقيق در جدول إخلاصه شده است.

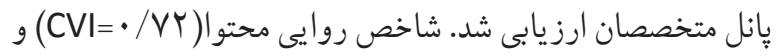

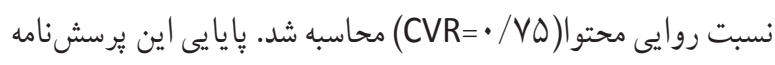

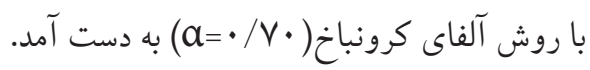
ير سش نامه دوم، برسش نامه سنجش فعاليت بدنى ساكنان بود،

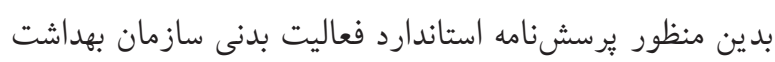

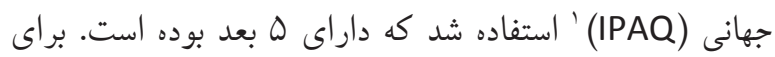
اين تحقيق از نسخه فارسى و استاندارد شده توسط واشقانى و

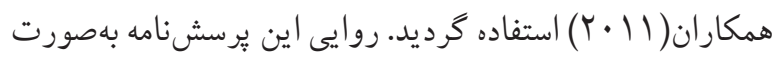

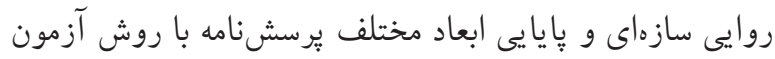

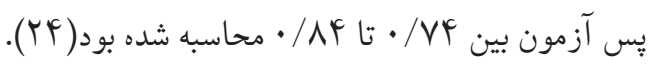

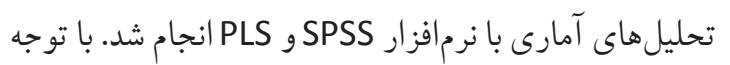

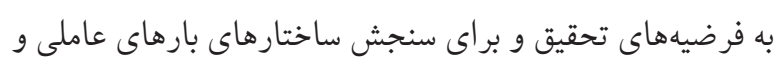
همبستكىهاى متقابل متغيرهاى تحقيق از آزمون حداقل مربعات

جدول ا. ارزيابى روايى و يايايى يرسشنامه تحقيق

\begin{tabular}{|c|c|c|c|c|}
\hline $\begin{array}{c}\text { ضر يب آلفاى كرونباخ } \\
\text { (Cronbach's a) }\end{array}$ & ميانكين واريانس تبيين شده & $\begin{array}{l}\text { ميانكين بار عاملى استاندارد } \\
\text { (SFL Mean) }\end{array}$ & متغير ها & \\
\hline . /VaF & $\cdot / 9 \mid F$ &.$/ 045$ & تنوع & \multirow{7}{*}{$\begin{array}{l}3 \\
3 \\
3 \\
3 \\
3 \\
3 \\
\overline{3} \\
\overline{2} \\
\bar{y}\end{array}$} \\
\hline$\cdot / \mathrm{VF}^{\mathrm{C}}$ & . $/ 0 T_{S}$ &.$/ 014$ & نظم & \\
\hline$\cdot / V S P$ & . Vצr & $\cdot / V \mu q$ & باز بودن فضا & \\
\hline$\cdot / \mathrm{A} \cdot \mathrm{r}$ &.$/ O F S$ & . /OrS & حضور عناصر طبيعى & \\
\hline$\cdot / \mathrm{VS}$ & ./V१५ & ./VTr & نكهدارى و پايش & \\
\hline 1 & 1 & 1 & ارزش تاريخى & \\
\hline - VFA & . $/ \Delta T^{\mu}$ & . Ars & سرزندگى & \\
\hline.$/ \mu \mu v$ & . $/ O Y T F^{\prime}$ & $\cdot 10 \cdot 4$ & فعاليت بدنى & \\
\hline$\cdot / \mathrm{V} \cdot \Lambda$ & - & - & 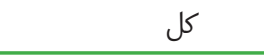 & \\
\hline
\end{tabular}

يافته ها

از مشار كت كنند گان را تشكيل دادهاند.

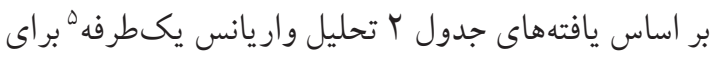
آزمون تفاوت در فعاليت بدنى ساكنين بر مبناى سن، تحصيلات

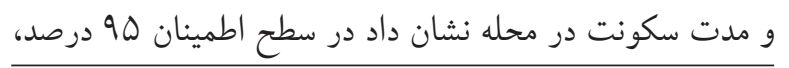

2. Partial Least Squares

3. Average Variance Extracted

4. Value Eigen

5. ANOVA
تحليل يافتهاى دمو گر افيك جامعه يُّوهش نشان داد كه FN

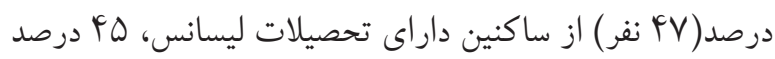

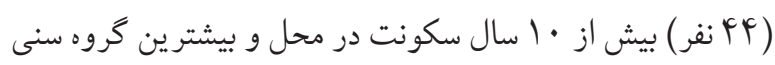

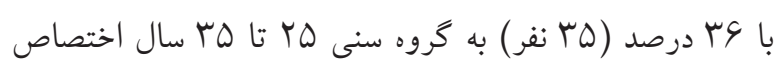

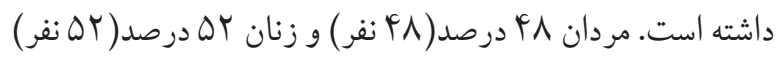

1. International Physical Activity Questionnaire(IPAQ) 


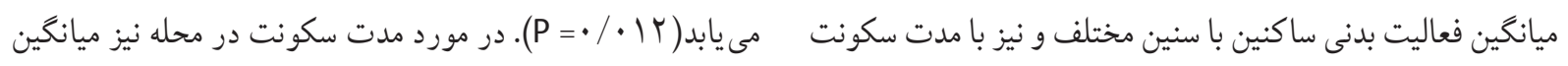

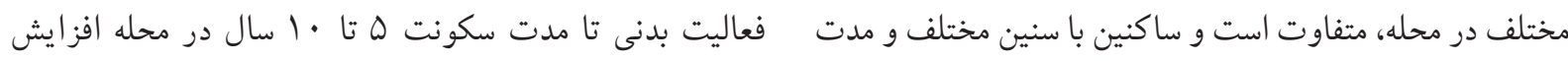

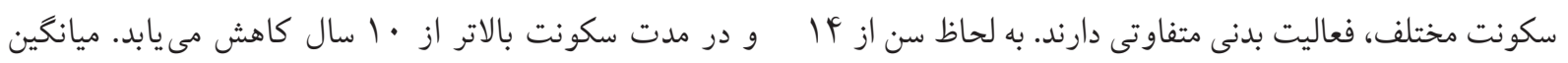

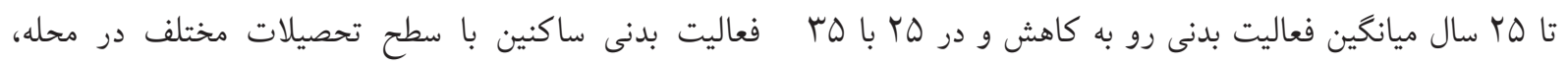

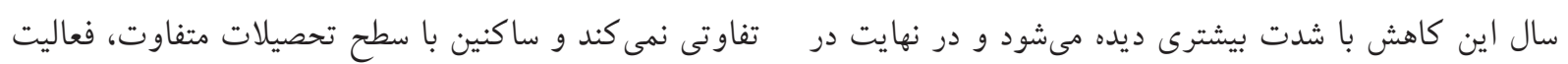

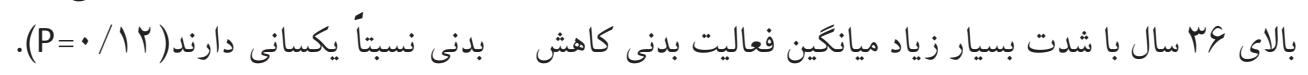

جدول r. نتايج ANOVA براى آزمون تفاوت در فعاليت بدنى بر مبناى سن، تحصيلات و مدت سكونت ساكنين در محله

\begin{tabular}{|c|c|c|c|c|}
\hline $\mathbf{F}$ & مقدار خطا & سطح معنادارى & متغير جمعيت شناختى & متغير وابسته \\
\hline F/r & $\cdot / \cdot \Delta$ &.$/ \cdot 1 r$ & سن & \multirow{3}{*}{ فعاليت بدنى } \\
\hline$r /|f|$ &.$/ .0$ &.$/ I T F$ & تحصيلات & \\
\hline$r / r s V$ & $\cdot / \cdot \Delta$ &.$/ \cdot r \Delta$ & مدت حضور يا سكونت & \\
\hline
\end{tabular}

بر اساس يافتهاى جدول لا، در ارتباط باكيفيت زيبايى شناختى شامل تنوع، نظم، باز بودن فضا، حضور عناصر طبيعى، نغهدارى و

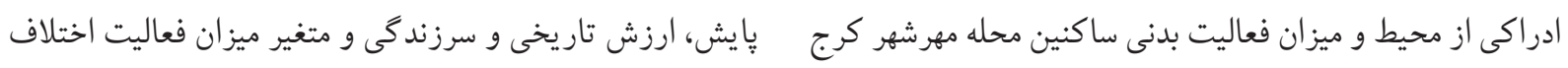

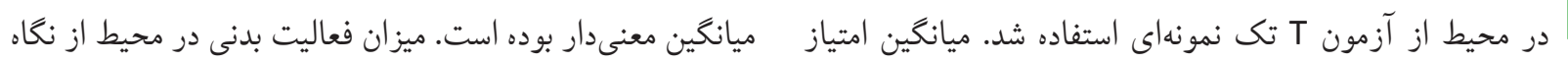

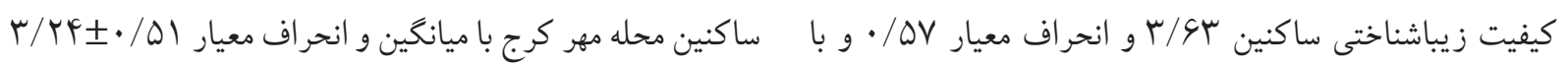

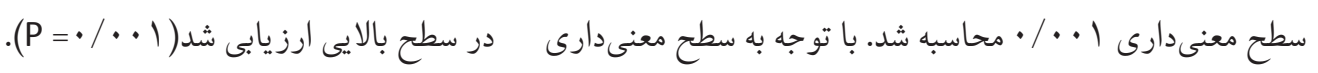

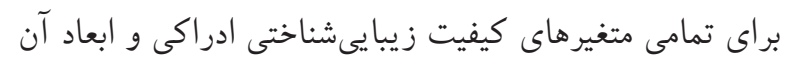

جدول س. آزمون T تك نمونهاى براى تبيين وضعيت كيفيت بصرى محيط و ابعادش

\begin{tabular}{|c|c|c|c|c|c|c|}
\hline \multicolumn{2}{|c|}{ حدود اختلافها در اطمينان ه 9\%٪ } & \multirow{2}{*}{ سطح معنادارى } & \multirow{2}{*}{ 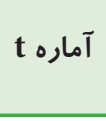 } & \multirow{2}{*}{ ميانغين و انحراف } & \multirow{2}{*}{ متغير } & \\
\hline 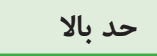 & حد يايين & & & & & \\
\hline$\cdot / V \Delta V$ &.$/ 010$ & $\cdot / \cdot \cdot 1$ & $\mid r / 1$ & $r / \cdot \pm G r / \Delta V$ & كيفيت زيبايىشناختى ادراكى & \\
\hline$\cdot / r \Delta r$ & $\cdot / \mu \cdot \Delta$ & $\cdot / \cdot \cdot 1$ & $\varphi / \wedge \Delta$ & $r / \cdot \pm F \Delta / \Delta \varphi$ & 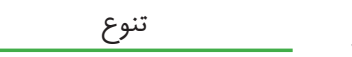 & \\
\hline$\cdot /$ ATF & $\cdot / F F \wedge$ & $\cdot / \cdots 1$ & $r / 9 \wedge$ & $r / \cdot \pm r q / v r$ & نظم & \\
\hline$\cdot /$ THA & $\cdot / 9 \cdot 1$ & $\cdot / \cdot 1$ & $9 / \mathrm{V} \Delta$ & $r / \cdot \pm \wedge 9 \cdot / \Delta \varphi$ & باز بودن فضا & \\
\hline$\cdot / r \mu r$ & $\cdot / 9 \cdot 1$ & $\cdot / \cdot 1$ & $11 / 9 \mu$ & $\boldsymbol{c} / \cdot \pm \cdot \Delta \cdot / \mu 1$ & حضور عناصر طبيعى & \\
\hline - /var & 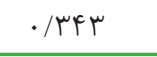 & $\cdot / \cdots 1$ & $\mid r / F F$ & $\mu / \cdot \pm 9 \Lambda \cdot / 9 V$ & نكهدارى و پايش & \\
\hline$-\cdot 1199$ & $-\cdot / 1 \& q$ & $\cdot / \cdots 1$ & $-r / 9 \Lambda$ & $r / \cdot \pm q r \cdot / 4 r$ & ارزش تاريخى & \\
\hline$\cdot / \vee \& 9$ & $\cdot / 0 \cdot \mu$ & $\cdot / \cdot 1$ & $q / r q$ & $\mu / \pm 9 \Lambda \cdot / 9 \mu$ & 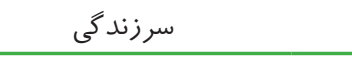 & \\
\hline$\cdot$ /VFr & $\cdot / \cdots 1$ & $\cdot / \cdots 1$ & $\Delta / V I$ & $r / \cdot \pm r r \cdot / \Delta l$ & فعاليت بدنى & \\
\hline
\end{tabular}

بر اساس يافتهاى جدول f أبراى بررسى ضرايب همبستگى نتايج نشان داد كه همبستگى تمامى متغيرهاى فرضيات تحقيق در

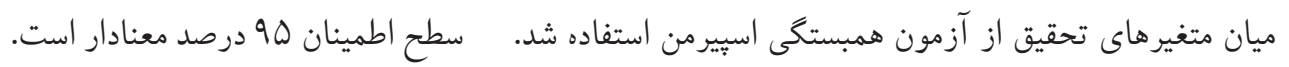


جدول F أ. ضرايب همبستكى ميان متغيرها

\begin{tabular}{|c|c|c|c|c|c|c|c|c|}
\hline فعاليت بدنى & سرزندگى & ارزش تاريخى & نَهايش & حضور عناصر & باز بودن فضا & نظم & تنوع & \\
\hline & & & & & & & $1 / \ldots$. 椪 & \multirow{2}{*}{ تنوع } \\
\hline & & & & & & & $\cdot / \cdots 1$ & \\
\hline & & & & & & $1 / \ldots$ 粰 & 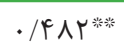 & \multirow{2}{*}{ نظم } \\
\hline & & & & & &.$/ .1$ &.$/ \ldots 1$ & \\
\hline & & & & & $1 / \ldots$ 料棌 & $\cdot / \mathbb{F} G^{\text {粕 }}$ & . / & \multirow{2}{*}{ باز بودن فضا } \\
\hline & & & & &.$/ . .1$ &.$/ \cdots 1$ & $\cdot / \cdot 0$ & \\
\hline & & & & 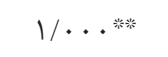 & $\cdot / r Y V^{*}$ & ( & 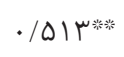 & \multirow{2}{*}{ حضور عناصر طبيعى } \\
\hline & & & &.$/ . .1$ & $\cdot / \cdot \Delta$ &.$/ \cdots$ &.$/ .1$ & \\
\hline & & & $1 / \ldots$ 䊪 & $\left.\cdot|\mathbb{A}|\right|^{\text {米䅴 }}$ & $\cdot / \mathcal{F} \Delta r^{* * * * *}$ & $\cdot / \Delta \wedge 9^{*}$ & $\cdot / \mathcal{F} \cdot V^{* * * * *}$ & \multirow{2}{*}{ نگهدارى و پايش } \\
\hline & & & $\cdot / \cdots 1$ & $\cdot / \cdots 1$ & $\cdot / \cdots 1$ & $\cdot 1 \cdot 0$ & $\cdot / \cdots 1$ & \\
\hline & & $1 / \ldots$ 粰 & 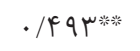 &.$/ . \times 9 *$ & 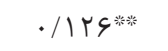 & 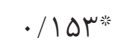 &.$/ \cdot 11^{*}$ & \multirow{2}{*}{ ارزش تاريخى } \\
\hline & &.$/ \cdot 1$ &.$/ .1$ & $\cdot / \cdot 0$ &.$/ \cdot 1$ & $\cdot / \cdot \Delta$ & $\cdot / \cdot \Delta$ & \\
\hline & $1 / \ldots$ ***** $^{*}$ & $\cdot / 1 \wedge \varsigma^{*}$ & $\cdot / \mu \cdot \boldsymbol{r}^{\mathrm{c} * * * *}$ & $\cdot / \mathcal{F} V V^{\text {米粰 }}$ & $\cdot / \cdot 19^{\text {䄅莎 }}$ & 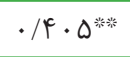 & $\cdot / \mu \cdot 9^{\text {****⿲二丨匕 }}$ & \multirow{2}{*}{ سرزندگى } \\
\hline &.$/ \cdots 1$ & $\cdot / \cdots 0$ &.$/ . .1$ &.$/ . .1$ & $\cdot, \cdot r$ &.$/ .1$ &.$/ . .1$ & \\
\hline $1 / \ldots$ 絖 & 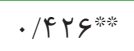 &.$/ / \mu \Delta *$ & $\cdot / \mathbb{F} \mid V^{* * * *}$ & 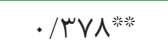 & $. / 1 F . *$ &.$/ \Delta F q^{* * * * * *}$ & $\cdot / \mathcal{F} \mathcal{F}^{\mathcal{*} * * *}$ & \multirow{2}{*}{ فعاليت بدنى } \\
\hline.$/ \ldots 1$ &.$/ \ldots 1$ & $\cdot / \cdots \Delta$ &.$/ \ldots 1$ &.$/ \cdots 1$ & $\cdot / \cdot \Delta$ &.$/ \ldots 1$ &.$/ \ldots 1$ & \\
\hline
\end{tabular}



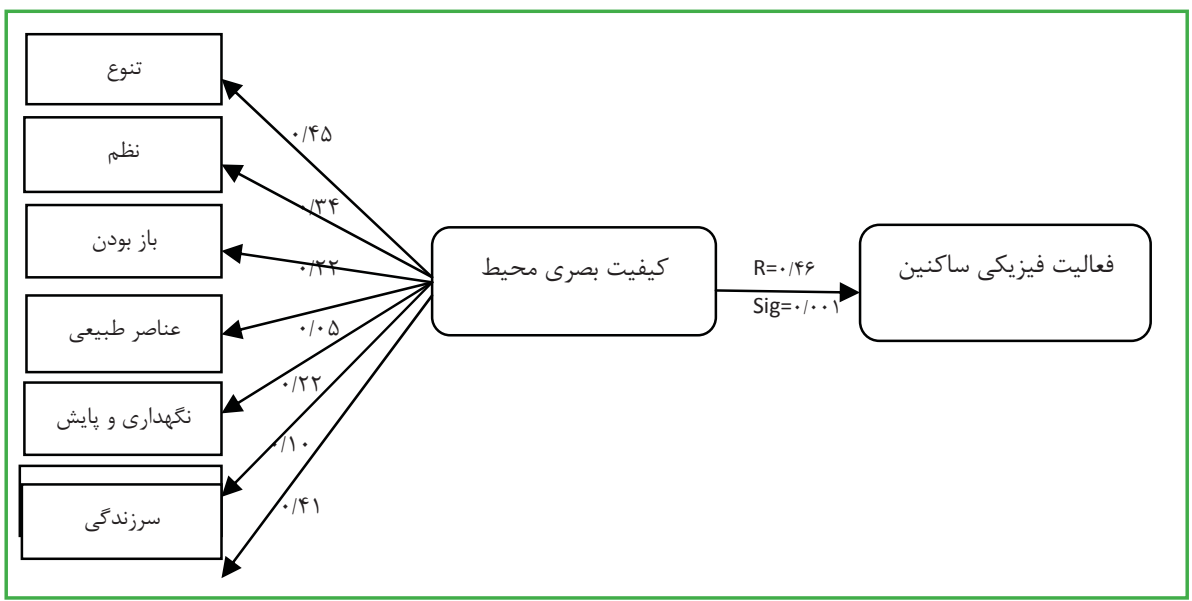

شكل ا. مدل تحقيق در حالت تخمين ضرايب مسير

بهمنظور تعيين تأثير كيفيت زيبايىشناختى ادراكى بر فعاليت محيط تأثير مثبت و معنادارى بر ميزان فعاليت بدنى ساكنين در

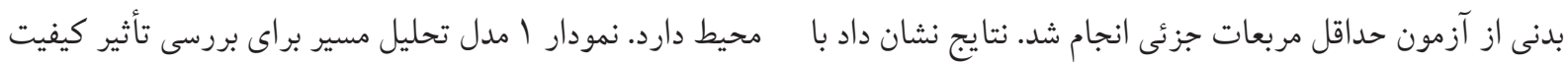

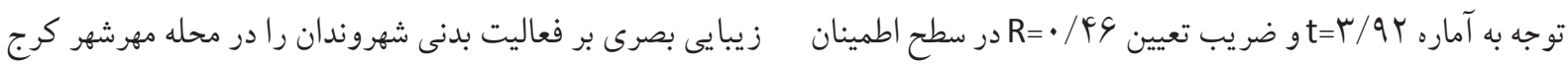
هو 99 درصد مورد كيفيت زيبايى بصرى ادراكى ساكنين از نشان دادهاست. 


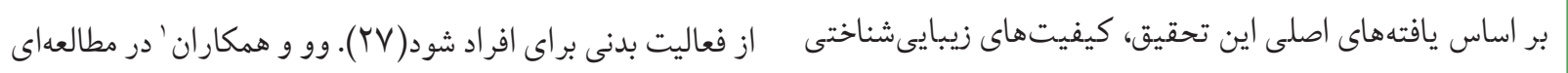

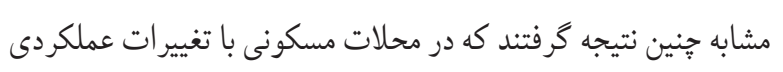
و ظاهر بصرى محلات، عملكرد سلامتى و ارتقاى سلامت بهتر است.

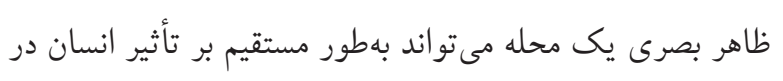

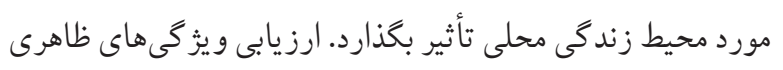

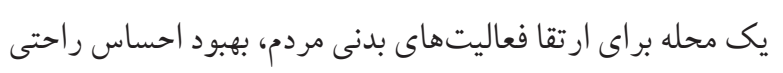

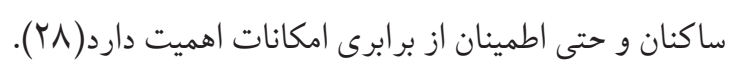
نتايج نشان داد كه فعاليت هاى بدنى به قصد رفت و آمد بيشتر

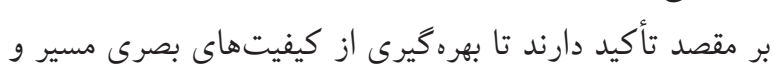

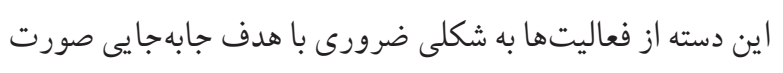

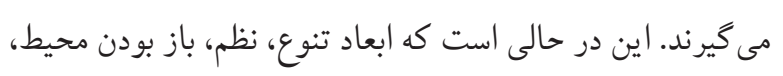

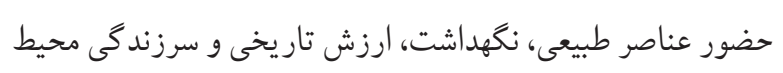
تأثير معنىدارى بر ميزان فعاليت بدنى به منظور فراغت و تفريح

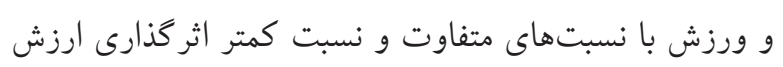

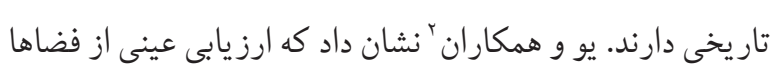

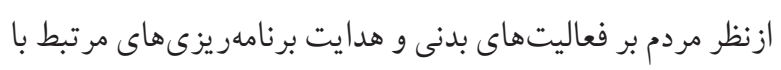

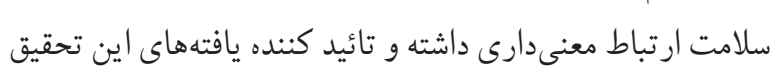

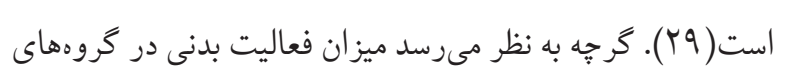

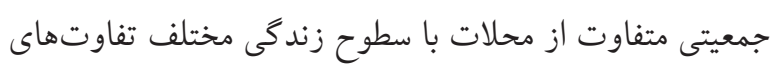

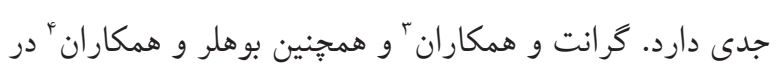

تحقيقات خويش نتايج مشابهى را يافتهاند( • بر, آس).

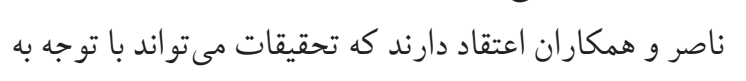
دانش فعلى درى محيطى براى كشف اقدامات و روشهاى مربوط

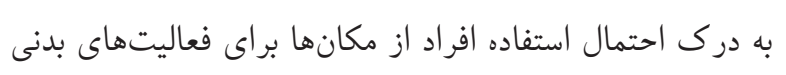

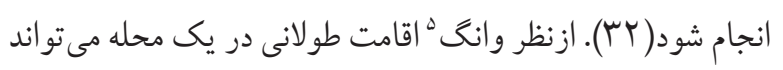

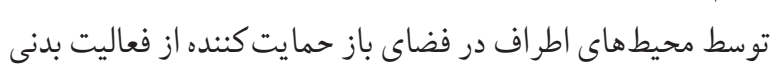

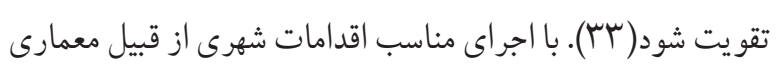

1. Wu C.

2. YuX.

3. Grant SJ etal.

4. Buehler R etal.

5. Wange $Z$ etal.

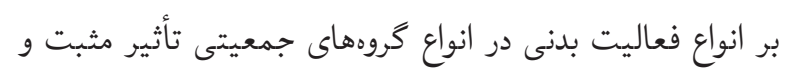
معنادارى بر ميزان فعاليت بدنى ساكنين در محيط دارد. نتايج

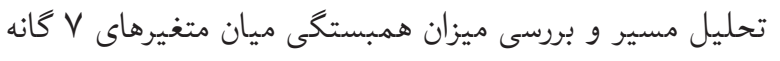

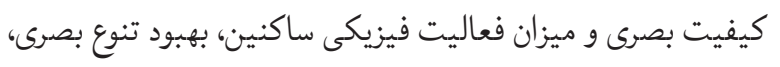
منظم تر شدن، بازتر و وسيع تر شدن محيط، طبيعى تر بودن، حفظ و و نكهاري بهتر، وجود عناصر تاريخى در محيط و شاداب و سرزنده بودن

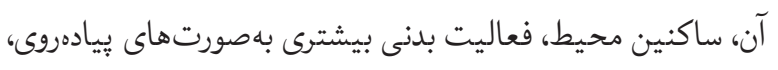

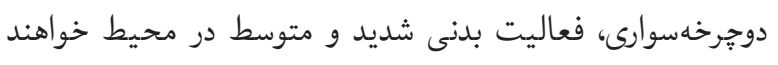

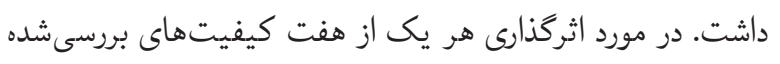

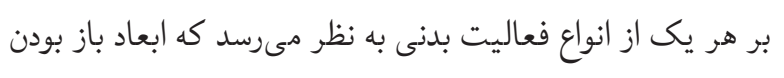

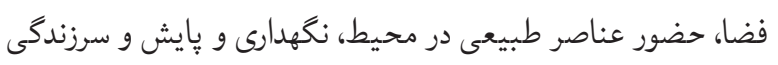

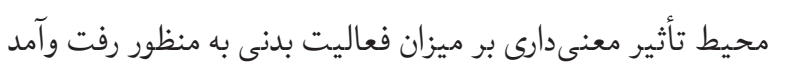

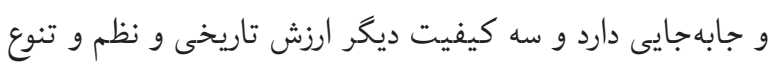

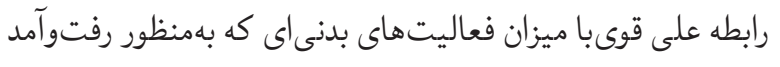
صورت مى گيرد، ندارد. فتحى و همكاران( · · · (Y) ضمن تائيد يافتهاى تحقيق ما نشان داد كه كيفيت محيطهاى ساخته شده نقش مهمى در كاهش اختلالات

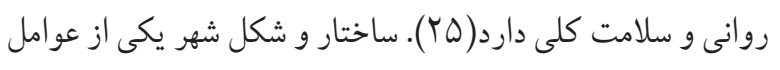

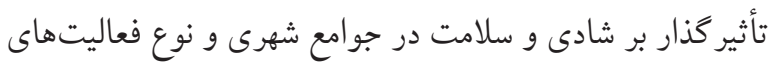
روزمره شهروندان محسوب مى شود. شكل شناسى شهر بايد شهروندان

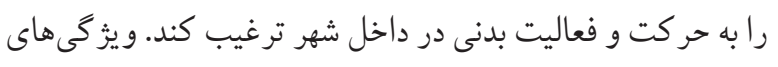
عملكردى، فيزيكى، فرهنكى اجتماعى و ادراكى تصويرى مهم ترين

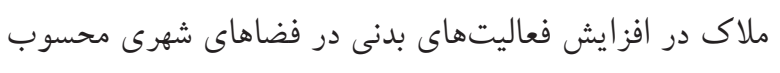

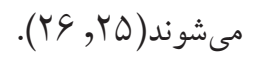
كراويس در تحقيقى نشان داد كه مداخلات طر احى در محيطهاى شهرى مى تواند فعاليت فيزيكى را در داخل و خارج از محلات

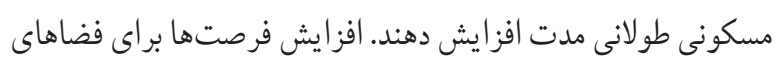

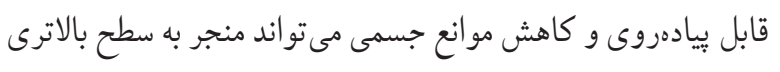


كرج انجام شد محدوديتهايى در برداشت. عدم همكارى براى تكميل سوالات يُرسشنامه و محدوديت در همكارى و كذاشتن

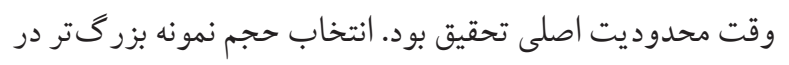

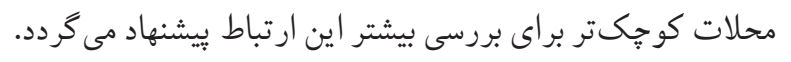

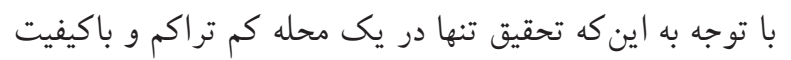
بصرى نسبتاً مطلوب آزموده شده است، در جهت تعميم بيشتر نتايج حاصل از آن، سنجش آن بر روى محلات مسكونى با ويز گیىهاى

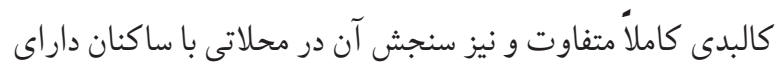

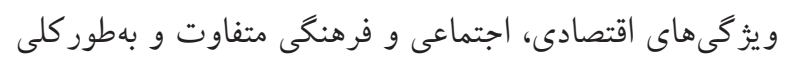

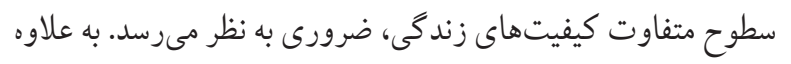

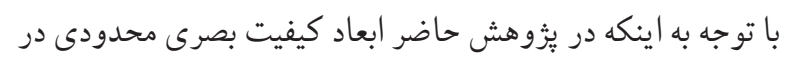
نظر گرفته شده است، با وارد نمودن ساير ابعاد كيفيت بصرى محلات مسكونى ممكن است نتايج متفاوتى حاصل كردد.

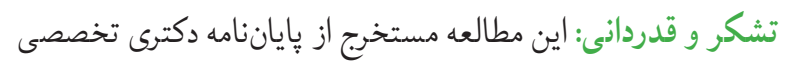

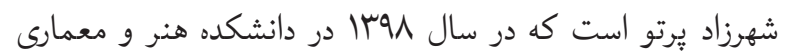
دانشعاه بوعلى سينا همدان تصويب شده است.

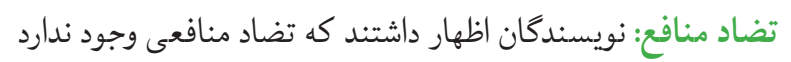

\section{References}

1. Samvat T, Esanejad A. National program of physical activity to promote health in the Islamic Republic of Iran. Tehran: Ministry of health and medical education; 2016.

2. Vahedian-Shahroodi M, Tehrani H, Robat-Sarpooshi D, GHolian-Aval M, Jafari A, Alizadeh-Siuki H. The impact of healtheducationonnutritionalbehaviorsinfemalestudents: An application of health belief model. International Journal of Health Promotion and Education. 2021;59(2):70-82. https://doi.org/10.1080/14635240.2019.1696219

3. Motamedi M, Peyman N, Afzalaghaee M. Rrelationship of health literacy and regular physical activity self-efficacy with body mass index in adolescent girls aged $15-18$ years. Journal of Health Literacy. 2020;5(3):64-73.

4. Wang J, Cao X. Exploring built environment correlates of walking distance of transit egress in the Twin Cities. Journal of transport geography. 2017;64:132-8. https://doi.org/10.1016/j.jtrangeo.2017.08.013

5. Jassemi Zergani $M$, Seirafi M-R, Taghdisi M-H, Malihi zuckerini S, Taghavi-Kojeidi H. Evaluation of effectiveness
شهرى و طر احى فضاها باز عمومى مز اياى قابل توجهى در ارتباط با

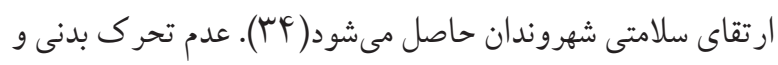

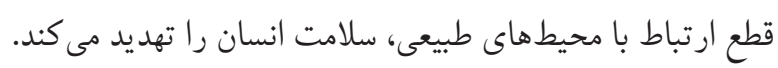
با اين حال تحقيقات نشان داده است كه محيطهاى طبيعى به طور

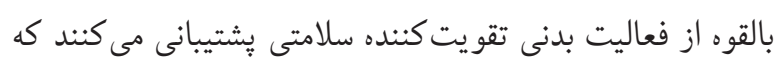

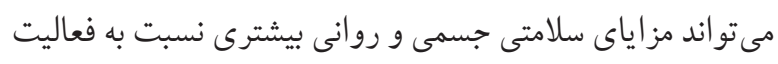

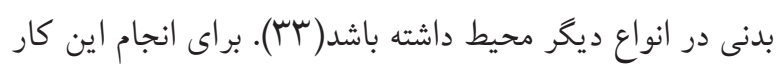

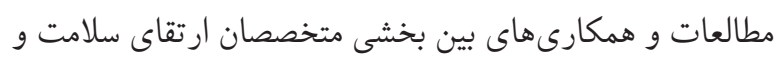

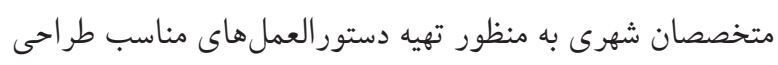
شهرى سلامت محور براى تصميم گيران و برنامهريزان و طر احان شهرى و متخصصين حوزه سلامت عمومى ضرورى است. مطالعات ارزيابى ويز گى هاى فضايى ظاهر بصرى و تأثير ان بر سلامتى هنوز محدود است. يافتهاى اكتشافى اين مقاله امكان بهبود مطالعات مربوط به كيفيت فضايى در مقياس انسانى را فراهم مى كند و ودان

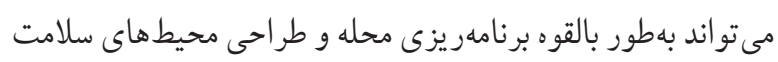
محور را تسهيل نمايد.

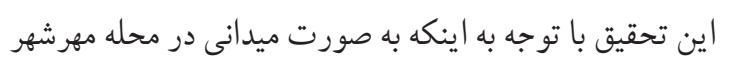

of integration of Mindfulness-Based-Eating Awareness Training and implementation intention model on Body Mass Index, Waist circumference, Mindfulness Eating, and Physical Activity in Obese Women. Iranian Journal of Health Education and Health Promotion. 2021;9(1):94-109.. https://doi.org/10.52547/ijhehp.9.1.94

6. Almeida OP, Khan KM, Hankey GJ, Yeap BB, Golledge J, Flicker L. 150 minutes of vigorous physical activity per week predicts survival and successful ageing: a populationbased 11-year longitudinal study of 12201 older Australian men. British journal of sports medicine. 2014;48(3):220-5. https://doi.org/10.1136/bjsports-2013-092814

7. Mahdifar M, Tavakoly sany SB, Ghavami V, VahedianShahroodi M. Health Literacy and Body Image Concern Among Healthcare Employees in Binaloud, Iran. Journal of Health Literacy. 2021;6(1):31-40.

8. Goodarzi A, Elahi AR, Akbari Yazdi H. Qualitative analysis of elderly physical activities development strategies and policies of selected and leading countries. Iranian Journal of 
HealthEducation and Health Promotion. 2020;8(4):359-77.. https://doi.org/10.29252/ijhehp.8.4.359

9. Sallis JF, Floyd MF, Rodríguez DA, Saelens BE. Role of built environments in physical activity, obesity, and cardiovascular disease. Circulation. 2012;125(5):729-37. https://doi.org/10.1161/CIRCULATIONAHA.110.969022

10. Giles-Corti B, Broomhall MH, Knuiman M, Collins C, Douglas $\mathrm{K}, \mathrm{Ng} \mathrm{K}$, et al. Increasing walking: how important is distance to, attractiveness, and size of public open space? American journal of preventive medicine. 2005;28(2):169-76. https://doi.org/10.1016/j.amepre.2004.10.018

11. Bentley R, Jolley D, Kavanagh AM. Local environments as determinants of walking in Melbourne, Australia. Social science \& medicine. 2010;70(11):1806-15. https://doi.org/10.1016/j.socscimed.2010.01.041

12. Parra DC, Gomez LF, Fleischer NL, Pinzon JD. Built environment characteristics and perceived active park use among older adults: Results from a multilevel study in Bogota. Health \& Place. 2010;16(6):1174-81. https://doi.org/10.1016/j.healthplace.2010.07.008

13. Bedimo-Rung AL, Mowen AJ, Cohen DA. The significance of parks to physical activity and public health: a conceptual model. American journal of preventive medicine. 2005;28(2):159-68. https://doi.org/10.1016/j.amepre.2004.10.024

14. Cervero R. Alternative approaches to modeling the travel-demand impacts of smart growth. Journal of the American Planning Association. 2006;72(3):285-95. https://doi.org/10.1080/01944360608976751

15. Lee $\mathrm{H}$, Kang H-M, Ko Y-J, Kim H-S, Kim Y-J, Bae $W$, et al. Influence of urban neighbourhood environment on physical activity and obesity-related diseases. Public health. 2015;129(9):1204-10. https://doi.org/10.1016/j.puhe.2015.06.002

16. Sugiyama T, Neuhaus M, Cole R, Giles-Corti B, Owen N. Destination and route attributes associated with adults' walking: a review. Medicine and science in sports and exercise. 2012;44(7):1275-86. https://doi.org/10.1249/MSS.0b013e318247d286

17. Rezvanipour S, Hassan N, Ghaffarianhoseini A, Danaee $M$. Why does the perception of street matter? A dimensional analysis of multisensory social and physical attributes shaping the perception of streets. Architectural Science Review. 2021:1-15. https://doi.org/10.1080/00038628.2020.1867818

18. Zhang $\mathrm{H}$, Lin $\mathrm{S}-\mathrm{H}$. Affective appraisal of residents and visual elements in the neighborhood: $A$ case study in an established suburban community.
Landscape and Urban Planning. 2011;101(1):11-21. https://doi.org/10.1016/j.landurbplan.2010.12.010

19. Parto S, Izadi Ms, Karimimoshaver M, Zaboli R. Urban Open Spaces Supporting Physical Activity and Promoting Citizen's Health: A Systematic Review. Iranian Journal of Health Education and Health Promotion. 2019;7(2):126-42. https://doi.org/10.30699/ijhehp.7.2.126

20. Topal HF, Hunt DV, Rogers CD. Exploring Urban Sustainability UnderstandingandBehaviour:ASystematicReviewtowards a Conceptual Framework. Sustainability. 2021;13(3):1139. https://doi.org/10.3390/su13031139

21. Reyes-Riveros R, Altamirano A, De La Barrera $F$, Rozas D, Vieli L, Meli P. Linking public urban green spaces and human well-being: A systematic review. Urban Forestry \& Urban Greening. 2021:127105. https://doi.org/10.1016/j.ufug.2021.127105

22. Doğan U. Examining Urban Design Characteristics of City Centers Using Walkability Criteria: Case of Turkey. Journal of Urban Planning and Development. 2021;147(2):04021003. https://doi.org/10.1061/(ASCE)UP.1943-5444.0000663

23. MacCallum RC, Widaman KF, Zhang S, Hong S. Sample size in factor analysis. Psychological methods. 1999;4(1):84. https://doi.org/10.1037/1082-989X.4.1.84

24. Vasheghani-Farahani A, Tahmasbi $M$, Asheri $H$, Ashraf $H$, Nedjat S, Kordi R. The Persian, last 7-day, long form of the International Physical Activity Questionnaire: translation and validation study. Asian journal of sports medicine. 2011;2(2):106. https://doi.org/10.5812/asjsm.34781

25. Fathi S, Sajadzadeh $H$, Mohammadi Sheshkal $F$, Aram F, Pinter G, Felde I, et al. The role of urban morphology design on enhancing physical activity and public health. International journal of environmental research and public health. 2020;17(7):2359. https://doi.org/10.3390/ijerph17072359

26. Gobster PH, Ribe RG, Palmer JF. Themes and trends in visual assessment research: Introduction to the Landscape and Urban Planning special collection on the visual assessment of landscapes. Landscape and Urban Planning. 2019;191:103635. https://doi.org/10.1016/j.landurbplan.2019.103635

27. Gharaveis A. A systematic framework for understanding environmental design influences on physical activity in the elderly population: A review of literature. Facilities. 2020;38(9-10):625-49. https://doi.org/10.1108/F-08-2018-0094

28. Wu C, Peng N, Ma X, Li S, Rao J. Assessing multiscale visual appearance characteristics of neighbourhoods 
using geographically weighted principal component analysis in Shenzhen, China. Computers, Environment and Urban Systems. 2020;84:101547. https://doi.org/10.1016/j.compenvurbsys.2020.101547

29. Yu X, Huo W, Niu B, Zhao M. Measuring Greenery Supportiveness in Park for Physical Activity Using Smartphone Panoramic Image and a Green Vegetation Extraction Index. Research Square; 2020. https://doi.org/10.21203/rs.3.rs-45628/v1

30. Buehler R, Pucher J, Bauman A. Physical activity from walking and cycling for daily travel in the United States, 2001-2017: Demographic, socioeconomic, and geographic variation. Journal of Transport \& Health. 2020;16:100811. https://doi.org/10.1016/j.jth.2019.100811

31. Grant SJ, Lithopoulos A, Rhodes RE. Understanding action control of physical activity among mothers with young children. International Journal of Sport and Exercise Psychology. 2021:1-17. https://doi.org/10.1080/1612197X.2020.1869805

32. Nasar JL. Assessing perceptions of environments for active living. American journal of preventive medicine. 2008;34(4):357-63. https://doi.org/10.1016/j.amepre.2008.01.013

33. Wang Z, Shepley MM. Can aging-in-place be promoted by the built environment near home for physical activity: a case study of non-Hispanic White elderly in Texas. Journal of Housing and the Built Environment. 2018;33(4):749-66. https://doi.org/10.1007/s10901-017-9584-z

34. Khan MN, Haq ZU, Khan M, Wali S, Baddia F, Rasul S, et al. Prevalence and determinants of violence against health care in the metropolitan city of Peshawar: a cross sectional study. BMC public health. 2021;21(1):1-11. https://doi.org/10.1186/s12889-021-10243-8 